\title{
A REFINEMENT OF THE POINCARÉ INEQUALITY FOR KOLMOGOROV OPERATORS ON $\mathbb{R}^{d}$
}

YASUHIRO FUJITA

Received 28 June 2004

We give a refinement of the Poincaré inequality for Kolmogorov operators on $\mathbb{R}^{d}$. This refinement yields some regularity result of the corresponding semigroups.

\section{Introduction}

Let $\left\{P_{t}\right\}$ be the semigroup on $B_{b}\left(\mathbb{R}^{d}\right)$ associated with the Kolmogorov operator

$$
L_{0}=\frac{1}{2} \Delta+F(x) \cdot D
$$

Here we denote by $B_{b}\left(\mathbb{R}^{d}\right)$ the Banach space of all Borel and bounded functions, endowed with the supremum norm. We assume a suitable dissipative assumption on the function $F=\left(F_{1}, \ldots, F_{d}\right)$ such that there exists a unique invariant probability measure $v$ on $\mathbb{R}^{d}$ associated with $\left\{P_{t}\right\}$. Let $H^{1}(\nu)$ and $H^{2}(\nu)$ be the Sobolev spaces with the norms

$$
\begin{aligned}
& \|\varphi\|_{H^{1}(v)}=\left[\int_{\mathbb{R}^{d}}\left[|\varphi|^{2}+|D \varphi|^{2}\right] d \nu\right]^{1 / 2}, \\
& \|\varphi\|_{H^{2}(v)}=\left[\int_{\mathbb{R}^{d}}\left[|\varphi|^{2}+|D \varphi|^{2}+\left|D^{2} \varphi\right|^{2}\right] d \nu\right]^{1 / 2},
\end{aligned}
$$

respectively. It is well known that the Poincaré inequality with respect to $v$ is the following:

$$
\int_{\mathbb{R}^{d}}(\varphi-\bar{\varphi})^{2} d \nu \leq \frac{1}{2 \alpha} \int_{\mathbb{R}^{d}}|D \varphi|^{2} d \nu, \quad \varphi \in H^{1}(\nu),
$$

where $\alpha>0$ is a constant determined by $F$, and $\bar{\varphi}=\int_{\mathbb{R}^{d}} \varphi d \nu$. The Poincaré inequality (1.4) is so important that it implies existence of a spectral gap or, equivalently, exponential 
convergence of equilibrium of the semigroup $\left\{P_{t}\right\}$ such that

$$
\int_{\mathbb{R}^{d}}\left|P_{t} \varphi-\bar{\varphi}\right|^{2} d \nu \leq e^{-2 \alpha t} \int_{\mathbb{R}^{d}}|\varphi|^{2} d \nu, \quad t \geq 0, \varphi \in L^{2}(\nu)
$$

(cf. [2, Proposition 3.12]).

The aim of this paper is to give a refinement of the Poincaré inequality (1.4) such that

$$
\int_{\mathbb{R}^{d}}(\varphi-\bar{\varphi})^{2} d \nu+\frac{1}{2 \alpha} \int_{0}^{\infty} d t \int_{\mathbb{R}^{d}}\left|D^{2} P_{t} \varphi\right|^{2} d \nu \leq \frac{1}{2 \alpha} \int_{\mathbb{R}^{d}}|D \varphi|^{2} d \nu, \quad \varphi \in H^{1}(\nu) .
$$

When $F(x)=-\alpha x$ in (1.1) (i.e., $\left\{P_{t}\right\}$ is the Ornstein-Uhlenbeck semigroup), inequality (1.6) is reduced to an equality. Furthermore, we will show that inequality (1.6) yields the regularity result such that $P_{t} \varphi-\bar{\varphi} \in L^{2}\left((0, \infty), d t ; H^{2}(\nu)\right)$ for $\varphi \in H^{1}(\nu)$. This regularity result corresponds to the well-known regularity result such that $P_{t} \varphi-\bar{\varphi} \in L^{2}((0, \infty)$, $\left.d t ; H^{1}(\nu)\right)$ for $\varphi \in L^{2}(\nu)$ (cf. (1.5) and (3.18)).

In the proof of the Poincaré inequality (1.4), the following inequality was used for $\varphi \in C_{b}^{1}\left(\mathbb{R}^{d}\right)$ :

$$
\left|D P_{t} \varphi(x)\right|^{2} \leq e^{-2 \alpha t} P_{t}\left(|D \varphi|^{2}\right)(x), \quad(t, x) \in(0, \infty) \times \mathbb{R}^{d}
$$

(cf. [2, Proposition 2.8]). In our proof of inequality (1.6), we will also use (1.7). However, we will derive another differential inequality so as not to lose the term $\left|D^{2} P_{t} \varphi(x)\right|^{2}$. For this purpose, it is crucial to assume that the Kolmogorov operator $L_{0}$ has the form of (1.1). It seems hard for the author to apply our proof directly to a more general Kolmogorov operator such as $(1 / 2) \operatorname{tr}\left[C(x) D^{2}\right]+F(x) \cdot D$.

The contents of this paper are as follows. In Section 2, we will state the main results. They will be proved in Section 3 .

\section{Main results}

First of all, we recall the results about invariant probability measures on $\mathbb{R}^{d}$ (for details, see [2]). Following [1, Hypothesis 1.1], we make the following assumptions on $F=\left(F_{1}, \ldots, F_{d}\right)$ of $(1.1)$.

(A) $F \in C^{4}\left(\mathbb{R}^{d} ; \mathbb{R}^{d}\right)$, and there exist

$$
\begin{gathered}
m \geq 0 \text { such that } \sup _{x \in \mathbb{R}^{d}} \frac{\left|D^{\beta} F(x)\right|}{1+|x|^{2 m+1-\beta}}<+\infty, \quad \beta=0,1,2,3,4, \\
\alpha>0 \text { such that } D F(x) y \cdot y \leq-\alpha|y|^{2}, \quad x, y \in \mathbb{R}^{d},
\end{gathered}
$$

$a, \gamma, c>0$ such that $(F(x+y)-F(x)) \cdot y \leq-a|y|^{2 m+2}+c\left(|x|^{\gamma}+1\right), \quad x, y \in \mathbb{R}^{d}$.

By [1, Proposition 1.2.2], the stochastic differential equation

$$
d \xi(t, x)=F(\xi(t, x)) d t+d w(t), \quad \xi(0, x)=x,
$$


admits a unique strong solution $(\xi(t, x))$, where $(w(t))$ is a $d$-dimensional standard Brownian motion on a probability space. Then we can define the semigroup $\left\{P_{t}\right\}$ on $B_{b}\left(\mathbb{R}^{d}\right)$ by

$$
P_{t} \varphi(x)=\mathbb{E}[\varphi(\xi(t, x))]
$$

By [2, Proposition 2.7], there exists a unique probability measure $\nu$ on $\mathbb{R}^{d}$ satisfying the following: for any uniformly continuous and bounded function $\chi$ on $\mathbb{R}^{d}$, we have

$$
\int_{\mathbb{R}^{d}} P_{t} \chi d \nu=\int_{\mathbb{R}^{d}} \chi d v, \quad t \geq 0
$$

Such a probability measure $\nu$ on $\mathbb{R}^{d}$ is called the invariant probability measure for $\left\{P_{t}\right\}$. Using this invariant probability measure $v$, we can extend $\left\{P_{t}\right\}$ to a strongly continuous semigroup of contractions on $L^{p}(\nu)$ for every $p \geq 1$. We also denote by $\left\{P_{t}\right\}$ this extended strongly continuous semigroup. The generator $\left(L, \operatorname{dom}_{p}(L)\right)$ of $\left\{P_{t}\right\}$ in $L^{p}(\nu)$ is the closure of the Kolmogorov operator $\left(L_{0}, C_{0}^{\infty}\left(\mathbb{R}^{d}\right)\right)$, where $L_{0}$ is the operator defined by $(1.1)$, and $C_{0}^{\infty}\left(\mathbb{R}^{d}\right)$ is the space of $C^{\infty}$-functions with compact supports. An important example of $L$ is the Ornstein-Uhlenbeck operator corresponding to the case $F(x)=-\alpha x$.

Next, we define the Sobolev spaces $H^{1}(\nu)$ and $H^{2}(\nu)$. The operators $\left(D, C_{0}^{\infty}\left(\mathbb{R}^{d}\right)\right)$ and $\left(D^{2}, C_{0}^{\infty}\left(\mathbb{R}^{d}\right)\right)$ are closable in $L^{p}(\nu)$ for every $p \geq 1$. We also denote their closures by $\left(D, \operatorname{dom}_{p}(D)\right)$ and $\left(D^{2}, \operatorname{dom}_{p}\left(D^{2}\right)\right)$, respectively. Then, we can define the Sobolev spaces $H^{1}(\nu)$ and $H^{2}(\nu)$ by $H^{1}(\nu)=\operatorname{dom}_{2}(D)$ and $H^{2}(\nu)=\operatorname{dom}_{2}\left(D^{2}\right)$, respectively. They become Hilbert spaces with the norms defined by (1.2) and (1.3), respectively. Then, the Poincaré inequality (1.4) holds for the constant $\alpha$ of (2.1).

Now, we state the main results of this paper.

Theorem 2.1. Assume (2.1). Then, for every $\varphi \in H^{1}(\nu)$,

$$
\begin{gathered}
P_{t} \varphi \in H^{2}(\nu), \quad \text { t-a.e. on }(0, \infty), \\
P_{t} \varphi-\bar{\varphi} \in L^{2}\left((0, \infty), d t ; H^{2}(\nu)\right), \\
\int_{\mathbb{R}^{d}}(\varphi-\bar{\varphi})^{2} d \nu+\frac{1}{2 \alpha} \int_{0}^{\infty} d t \int_{\mathbb{R}^{d}}\left|D^{2} P_{t} \varphi\right|^{2} d \nu \leq \frac{1}{2 \alpha} \int_{\mathbb{R}^{d}}|D \varphi|^{2} d \nu . \\
\text { When } F(x)=-\alpha x \text {, inequality (2.7) is reduced to an equality. }
\end{gathered}
$$

Results (2.5) and (2.6) give a regularity result of $P_{t} \varphi$ for $\varphi \in H^{1}(\nu)$. On the other hand, results (2.7) and (2.8) give refinements of the Poincaré inequality.

\section{Proof of Theorem 2.1}

In this section, we prove Theorem 2.1. For $\varphi \in C_{0}^{\infty}\left(\mathbb{R}^{d}\right)$, we set

$$
\eta(t, x)=P_{t} \varphi(x), \quad(t, x) \in[0, \infty) \times \mathbb{R}^{d} .
$$

First, we give two lemmas. 
Lemma 3.1. Assume (2.1). If $\varphi \in C_{0}^{\infty}\left(\mathbb{R}^{d}\right)$, then

$$
\begin{gathered}
(D \eta)_{t}, D^{\beta} \eta(\beta=0,1,2,3) \text { are continuous on }[0, \infty) \times \mathbb{R}^{d} \\
(D \eta)_{t}=D \eta_{t} \quad \text { on }[0, \infty) \times \mathbb{R}^{d}
\end{gathered}
$$

Proof. Since $F \in C^{4}\left(\mathbb{R}^{d} ; \mathbb{R}^{d}\right)$ and $\varphi \in C_{0}^{\infty}\left(\mathbb{R}^{d}\right)$, it follows from the theory in [1, Chapter 1] that

$$
D^{\beta} \eta \text { is continuous on }[0, \infty) \times \mathbb{R}^{d} \text { for } \beta=0,1,2,3 \text {. }
$$

Since $\eta$ of (3.1) satisfies the Kolmogorov equation

$$
\eta_{t}=\frac{1}{2} \Delta \eta+F \cdot D \eta \quad \text { on }[0, \infty) \times \mathbb{R}^{d}
$$

we have, for any $R, T>0$,

$$
D \eta(t+h, x)-D \eta(t, x)=\int_{t}^{t+h} D L \eta(s, x) d s, \quad 0 \leq t \leq T,|x|<R,
$$

where $h \in \mathbb{R}$ is chosen such that $t+h \geq 0$. By (3.4) and (3.6), we conclude that $D \eta(t, x)$ is differentiable with respect to $t$ for $|x|<R$ and

$$
(D \eta)_{t}(t, x)=D L \eta(t, x)=D \eta_{t}(t, x), \quad 0 \leq t \leq T,|x|<R .
$$

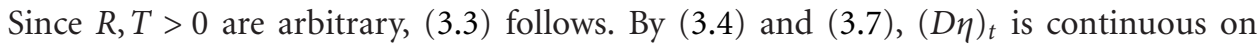
$[0, \infty) \times \mathbb{R}^{d}$. The proof is complete.

Lemma 3.2. Assume that (2.1) holds and $\varphi \in C_{0}^{\infty}\left(\mathbb{R}^{d}\right)$. Let

$$
\chi(t, x)=|D \eta(t, x)|^{2}=\sum_{j=1}^{d}\left|D_{j} \eta(t, x)\right|^{2}, \quad(t, x) \in[0, \infty) \times \mathbb{R}^{d} .
$$

Then,

$$
\begin{gathered}
\chi_{t}, D^{\beta} \chi(\beta=0,1,2) \text { are continuous on }[0, \infty) \times \mathbb{R}^{d}, \\
\left|D^{2} \eta\right|^{2}+\chi_{t} \leq L \chi-2 \alpha \chi \quad \text { on }[0, \infty) \times \mathbb{R}^{d} .
\end{gathered}
$$

When $F(x)=-\alpha x$, inequality (3.10) is reduced to an equality.

Proof. We obtain (3.9) from (3.2). Differentiating equation (3.5) with respect to $x_{j}$, we have, by (3.3),

$$
\left(D_{j} \eta\right)_{t}=\frac{1}{2} \Delta\left(D_{j} \eta\right)+\sum_{i=1}^{d} F_{i}\left[D_{i}\left(D_{j} \eta\right)\right]+\sum_{i=1}^{d}\left(D_{j} F_{i}\right)\left(D_{i} \eta\right) \quad \text { on }[0, \infty) \times \mathbb{R}^{d}
$$


On the other hand, we note that

$$
\begin{gathered}
\frac{1}{2} D_{i} \chi=\sum_{j=1}^{d}\left(D_{j} \eta\right)\left[D_{i}\left(D_{j} \eta\right)\right], \quad 1 \leq i \leq d, \\
\frac{1}{2} \Delta \chi=\left|D^{2} \eta\right|^{2}+\sum_{j=1}^{d}\left(D_{j} \eta\right)\left[\Delta\left(D_{j} \eta\right)\right], \\
\sum_{i, j=1}^{d}\left(D_{j} F_{i}\right)\left(D_{i} \eta\right)\left(D_{j} \eta\right) \leq-\alpha \chi .
\end{gathered}
$$

Here we used (2.1) in (3.14). Inequality (3.14) is reduced to an equality when $F(x)=-\alpha x$. Then, by (3.11)-(3.14), we obtain on $[0, \infty) \times \mathbb{R}^{d}$

$$
\begin{aligned}
\frac{1}{2} \chi_{t}= & \sum_{j=1}^{d}\left(D_{j} \eta\right)\left(D_{j} \eta\right)_{t} \\
= & \frac{1}{2} \sum_{j=1}^{d}\left(D_{j} \eta\right)\left[\Delta\left(D_{k} \eta\right)\right] \\
& +\sum_{i, j=1}^{d} F_{i}\left(D_{j} \eta\right)\left[D_{i}\left(D_{j} \eta\right)\right]+\sum_{i, j=1}^{d}\left(D_{j} F_{i}\right)\left(D_{i} \eta\right)\left(D_{j} \eta\right) \\
\leq & \frac{1}{2}\left(\frac{1}{2} \Delta \chi-\left|D^{2} \eta\right|^{2}\right)+\frac{1}{2} F \cdot D \chi-\alpha \chi
\end{aligned}
$$

Thus, (3.10) follows. It is easy to see that inequality (3.10) is reduced to an equality when $F(x)=-\alpha x$. The proof is complete.

Now, we prove Theorem 2.1.

\section{Proof of Theorem 2.1.}

Step 1. In this step, we will show Theorem 2.1 under the assumption that $\varphi \in C_{0}^{\infty}\left(\mathbb{R}^{d}\right)$. We choose $0<T<+\infty$ arbitrarily. Integrating (3.10) over $[0, T] \times \mathbb{R}^{d}$ with respect to $d t \times d \nu$, we have

$$
\begin{gathered}
\int_{0}^{T} d t \int_{\mathbb{R}^{d}}\left|D^{2} \eta(t, \cdot)\right|^{2} d \nu+\int_{\mathbb{R}^{d}}\left[|D \eta(T, \cdot)|^{2}-|D \varphi(\cdot)|^{2}\right] d \nu \\
\leq \int_{0}^{T} d t \int_{\mathbb{R}^{d}} L \chi(t, \cdot) d \nu-2 \alpha \int_{0}^{T} d t \int_{\mathbb{R}^{d}}|D \eta(t, \cdot)|^{2} d \nu .
\end{gathered}
$$

By Lemma 3.2, inequality (3.16) is reduced to an equality when $F(x)=-\alpha x$. Since $v$ is the invariant probability measure for $\left\{P_{t}\right\}$ as in (2.4), we have

$$
\int_{\mathbb{R}^{d}} L \chi(t, \cdot) d v=0, \quad t \geq 0
$$


On the other hand, by [2, Corollary 3.6], we have

$$
\int_{0}^{T} d t \int_{\mathbb{R}^{d}}\left|D P_{t} \varphi\right|^{2} d \nu=\int_{\mathbb{R}^{d}}\left[|\varphi|^{2}-\left|P_{T} \varphi\right|^{2}\right] d \nu
$$

Thus, we obtain by (3.16)-(3.18)

$$
\begin{gathered}
\int_{0}^{T} d t \int_{\mathbb{R}^{d}}\left|D^{2} P_{t} \varphi\right|^{2} d \nu+2 \alpha \int_{\mathbb{R}^{d}}\left[|\varphi|^{2}-\left|P_{T} \varphi\right|^{2}\right] d \nu \\
\leq \int_{\mathbb{R}^{d}}|D \varphi|^{2} d \nu-\int_{\mathbb{R}^{d}}\left|D P_{T} \varphi\right|^{2} d \nu, \quad T>0 .
\end{gathered}
$$

Now, let $T$ tend to positive infinity in (3.19). Using (1.7) and the ergodic property

$$
\lim _{T \rightarrow \infty} P_{T} \varphi(x)=\bar{\varphi}, \quad x \in \mathbb{R}^{d}
$$

(cf. [2, (3.11)]), we have obtained (2.7). Then, by (1.5) and (3.18), we have (2.5) and (2.6). Since inequality (3.19) is reduced to the equality when $F(x)=-\alpha x$, it is not difficult to see (2.8).

Step 2. In this step, we conclude Theorem 2.1. Let $\varphi \in H^{1}(\nu)$. Since $C_{0}^{\infty}\left(\mathbb{R}^{d}\right)$ is dense in $H^{1}(\nu)$, we can choose $\left\{\varphi_{n}\right\} \subset C_{0}^{\infty}\left(\mathbb{R}^{d}\right)$ such that $\varphi_{n} \rightarrow \varphi$ in $H^{1}(\nu)$. By Step 1 , we see that

$$
\int_{0}^{\infty} d t \int_{\mathbb{R}^{d}}\left|D^{2} P_{t} \varphi_{m}-D^{2} P_{t} \varphi_{n}\right|^{2} d \nu \leq \int_{\mathbb{R}^{d}}\left|D \varphi_{m}-D \varphi_{n}\right|^{2} d \nu
$$

Thus, $\left\{D^{2} P_{t} \varphi_{n}\right\}$ is a Cauchy sequence in $L^{2}\left((0, \infty) \times \mathbb{R}^{d}, d t \times d v ; \mathbb{R}^{d^{2}}\right)$. Hence, we find an element $f \in L^{2}\left((0, \infty) \times \mathbb{R}^{d}, d t \times d v ; \mathbb{R}^{d^{2}}\right)$ such that

$$
D^{2} P . \varphi_{n}(\cdot) \longrightarrow f(\cdot, \cdot) \quad \text { in } L^{2}\left((0, \infty) \times \mathbb{R}^{d}, d t \times d v ; \mathbb{R}^{d^{2}}\right)
$$

By the Fubini theorem, we see that $f(t, \cdot) \in L^{2}\left(\mathbb{R}^{d}, v ; \mathbb{R}^{d^{2}}\right), t$-a.e. On the other hand, by (3.22), we find a subsequence $\left\{n_{j}\right\}$ such that

$$
\int_{\mathbb{R}^{d}}\left|D^{2} P_{t} \varphi_{n_{j}}(\cdot) \longrightarrow f(t, \cdot)\right|^{2} d \nu \longrightarrow 0, \quad t \text {-a.e. }
$$

This means that

$$
D^{2} P_{t} \varphi_{n_{j}}(\cdot) \longrightarrow f(t, \cdot) \quad \text { in } L^{2}\left(\mathbb{R}^{d}, v ; \mathbb{R}^{d^{2}}\right), \quad t \text {-a.e. }
$$

Since $P_{t} \varphi_{n_{j}} \in H^{2}(\nu)\left(=\operatorname{dom}_{2}\left(D^{2}\right)\right)$ and $D^{2}$ is a closed operator in $L^{2}(\nu)$, we obtain

$$
P_{t} \varphi \in H^{2}(\nu), \quad f(t, \cdot)=D^{2} P_{t} \varphi(\cdot), \quad t \text {-a.e. }
$$


Then we obtain (2.5). Next, by (3.24), (3.25), Step 1, and Fatou's lemma, we have

$$
\begin{aligned}
\int_{0}^{\infty} d t \int_{\mathbb{R}^{d}}\left|D^{2} P_{t} \varphi\right|^{2} d \nu & \leq \liminf _{n \rightarrow \infty} \int_{0}^{\infty} d t \int_{\mathbb{R}^{d}}\left|D^{2} P_{t} \varphi_{n_{j}}\right|^{2} d \nu \\
& \leq \liminf _{n \rightarrow \infty} \int_{\mathbb{R}^{d}}\left|D \varphi_{n_{j}}\right|^{2} d \nu \\
& =\int_{\mathbb{R}^{d}}|D \varphi|^{2} d \nu
\end{aligned}
$$

Hence, by (1.5) and (3.18), we obtain (2.6). Finally, by (3.22) and (3.25), we conclude that

$$
D^{2} P . \varphi_{n}(\cdot) \longrightarrow D^{2} P . \varphi(\cdot) \quad \text { in } L^{2}\left((0, \infty) \times \mathbb{R}^{d}, d t \times d v ; \mathbb{R}^{d^{2}}\right) .
$$

Therefore, (2.7) follows from Step 1. By (3.27) and Step 1, it is easy to see (2.8). The proof is complete.

\section{References}

[1] S. Cerrai, Second Order PDE's in Finite and Infinite Dimension. A Probabilistic Approach, Lecture Notes in Mathematics, vol. 1762, Springer-Verlag, Berlin, 2001.

[2] G. Da Prato and B. Goldys, Elliptic operators on $\mathbb{R}^{d}$ with unbounded coefficients, J. Differential Equations 172 (2001), no. 2, 333-358, Erratum, J. Differential Equations, 184 (2002), no. 2, p. 620.

Yasuhiro Fujita: Department of Mathematics, Toyama University, Toyama 930-8555, Japan E-mail address: yfujita@sci.toyama-u.ac.jp 